\title{
Novel Multiresolution Metrics for Content-Based Image Retrieval
}

\author{
Zheng-Yun Zhuang, Ming Ouhyoung \\ wayne@cmlab.csie.ntu.edu.tw, ming@cmlab.csie.ntu.edu.tw \\ Communications and Multimedia Laboratory, Department of \\ Computer Science and Information Engineering, National Taiwan University, Taipei, Taiwan
}

\begin{abstract}
This paper proposes three new ideas and one revision about image metrics and their applications. Of most importance is the multiresolution 'shape metric', which measures distances according to images' content shape information. Accompanied with it is a feature/non-feature image characterization philosophy. The second effort is the modification of an existing 'color metric', whose distance computation is dominated by color distribution of images. The third idea is on analyzing image querying behavior of the general public, so as to propose and design a versatile 'power metric', which holds the properties of both the 'shape metric' and the 'color metric', for dealing with various kinds of hand-drawn queries. Finally, after applying the 'shape metric' to video shot boundary detection, a multiresolution scene change detection algorithm is proposed. For now, we have implemented these metrics on a personal computer for two applications, one is for image database management with content-based indexing and the other is for video shot boundary detection. Experiments show that the speed and the accuracy of both image retrieval and scene change detection are quite well.
\end{abstract}

\section{Introduction}

\subsection{Motivation and Objective}

As the need of data mining on the Web grows, various kinds of search engines are developed. Search engines responsible for keyword indexing are now available and the technology is reliable. Of course, one may use the same technology to built a search engine that is responsible for image searching by keywords. But if that were true, the search engine would be very difficult to use, due to the fact that properties of images may not be suitably coded. There is no standard rule in the world for image naming, Besides, different image producers may give different names to the same image.

If we examine on this problem more closely, we will find that a better way to find a particular image on the Web is to provide content-based image retrieval(or indexing) and to maintain such a database periodically with a spider program. To date, however, there are only a few content-based image database systems that have already been developed, let alone applications on the Web. Our goal is to develop a system that can be extended for content-based image search on the Web.

For our purpose, we design our image metrics with the feature extraction method in wavelet transform domain due to the fact that multiresolution analysis on images provides a good way to characterize images, to find the signature of images, and to catch the most significant information of them. Moreover, for properly designing these metrics, we had observed that in user hand-drawn image querying systems, a user may behave as an impressionist and emphasize on the color distribution in his query images while another one may like to give a sketch and draw the shape of her figure in monochrome. This makes us design our image metrics according to these two criteria. Therefore, the result includes three metrics. Shape metric computes the distance between images by their content shape information while color metric does by the color distribution information. Yet another one, the power metric, which is adjustable and is a mixture of the above two, counts on both kinds of information.

\subsection{Overview}

In section 2, we will have theoretical discussions about the proposed metrics. Image metric concepts and some metrics that were proposed previously are discussed in section 2.1 and 2.2. Then we give in brief the definitions of our metrics and explain how they operate in section 2.3 without telling why. Section 2.4 reveals some observations about the user behavior on producing a query image. Then section 2.5 explores the proposed metrics whereas 2.6 unveils the mechanism of the shape metric in more detail.

The system implementation of the proposed metrics is to be introduced in section 3 in short. Section 4 will show the experiments, benchmarks, and results about the met- 
rics and the system. After that, we will move to the conclusion, discussion, and future work in section 5 .

\section{II.Proposed Adjustable Metric}

\subsection{Overview}

To measure the distance between two images, we need a conceptual ruler or, a metric. For image search, the metric is used to compute the distance between the two characteristics of the query image and some target image in database. These distances are then sorted in order to form a queue that each queue member represents one image in database.

In our metric system, both the shape metric $L^{S}$ and the color metric $L^{C}$ are fixed. Only the power metric $L^{P}$, which is a mixture of the above two, behaves like a flexible amoeba and can be changed on demand. However, it copes with all kinds of hand-drawn query images.

\subsection{Existing Metrics}

Some previous image metrics are intuitive and straightforward. In the area of machine and robot vision, given two image planes $A$ and $B$, probably the most obvious metrics used to measure the distance or error between the two images are the pixel difference metrics. These metrics in image processing usually cost a lot of time, especially the $L 2$ metric.

Recent researchers solve these problems by some feature extraction techniques or some fast metrics[1][3][4] [5]. Some people find the similarity between images by histogram-based or DCT-intensity-based approaches. These might be suitable for those query images that were scanned but not so proper for hand-painted or drawn query. Others try to find a metric that counts primarily those types of differences that a human would use for discriminating images, but give less weight to the types of errors that a human might ignore for this task. Fortunately, such an image metric, associated with the multiresolution signal decomposition, has been proposed in [3] with their experiments in image querying. In the articles, that proposed metric is called the $L^{Q}$ metric. However, as we shall discuss, this metric deals with 'painted query' in major but may not cope with all the drawing behaviors of those who want to query.

\subsection{Proposed Metrics}

\subsubsection{Metric Definitions}

Conceptually, our metric $L^{P}$ is a dynamic mixture of $L^{S}$ and $L^{C}$, where $L^{S}$ is the ShapeMetric and $L^{C}$ the Color-
Metric. Mathematically and globally, our $L^{P}$ metric is defined as:

$L^{P}:\|A, B\|_{\rho}=\lambda\|A, B\|_{S}+(1-\lambda)\|A, B\|_{C}$

where $L^{S}$ is defined by:

$L^{s}:\|A, B\|_{S}=\sum_{i, j} s W_{i, j} A \operatorname{ssert}\left(A_{Y}^{\prime \prime}[i][j] \neq B_{Y}^{\prime \prime}[i][j]\right)$

or equally,

$\|A, B\|_{S}=\sum_{j, j} S W_{i, j}|| A^{\prime \prime}[i][j]|-| B^{\prime \prime}[i][j] \mid$

while $L^{C}$ is defined by:

$$
\begin{aligned}
L^{C}:\|A, B\|_{C} & =c W_{0,0}\left|A^{\prime}[0][0]-B^{\prime}[0][0]\right| \\
& +\sum_{i, j} c W_{i, j}\left|A^{\prime \prime}[i][j]-B^{\prime \prime}[i][j]\right|
\end{aligned}
$$

From the above definition, note that each one of $A^{\prime}$ and $B^{\prime}$ is some plane of the transform domain image (they are the transform domain images of source images $A$ and $B$ ). We will use the word plane, or channel, to represent different domains of an image rather than use the word domain directly to avoid the ambiguity. In our system, after we have performed multiresolution 2-D Haar wavelet decomposition on an image, we can get the transform domain image of it. For each image plane, the transform domain representation of it contains one $D C$ value in the upper-leftmost pixel(Plane[0][0]) and the other pixel values (Plane $[i][j]$ where $i, j \neq 0$ ) are treated as coefficients after transformation. These transform domain coefficients, together with the DC value, are more significant and may give more information to us than our source domain image pixels.

We adopted the standard Haar basis to be our decomposition basis for performing multiresolution wavelet transformation. Although Haar Transform has poor compression ratio in signal coding[7], it is very fast and has been proved to be a good image feature extraction transform[2][3]. As we know, two-dimensional Haar transform $(2-D H T)$ is an orthogonal transform and is separable.[7] This means that we can perform a 2-D Haar transform on a 2-D image in two phases, with each phase being composed of 1-D Haar transforms.

The second point worth mentioning is that both $A^{\prime \prime}$ and $A^{\prime \prime \prime}$ are the truncated and quantized version of $A^{\prime}$, which has been transformed, and so is it to $B^{\prime \prime}$ and $B^{\prime \prime \prime}$. What differs is the number of quantization levels. Coefficient values in $A^{\prime \prime}$ are of three quantization levels $(-1,0$, 1) for the computation of $L^{C}$ as was suggested in [3], whereas those in $A^{\prime \prime \prime}$ are of two levels $(0,1)$ for the computation of the $L^{S}$ metric, as we shall discuss in the next sub-section. Besides, only metric $L^{C}$ involves the DC value, which is proportional to the average density of the 
source image plane, in the original transform domain plane. $L^{\mathcal{S}}$ considers nothing about DC values.

Third, the shape metric $L^{S}$ is slightly different in context, but is very different in semantics from the color metric $L^{C}$, which is derived from the $L^{Q}$ that we had referred to. We will make this point clearer in section 2.6. The fourth and the last, our final $L^{P}$ is evidently a metric of mixture. Although there are no individual research outside our system made to prove the discriminating capability of the metric $L^{S}$, which is responsible for finding shape-like images, we can see the ability of this metric in our query experiments by setting the parameter $\lambda$ of $L^{P}$ metric and in our scene change detection experiment where the $L^{S}$ metric is applied.

\subsubsection{Two-Level Feature/Non-feature Quantization}

Here we list the quantization method mentioned in the previous discussion. The two-level quantization plane $A^{\prime \prime \prime}$ for the computation of $L^{S}$ is obtained from quantizing the transformed plane $A^{\prime}$ by the following formula.

$$
\begin{aligned}
& A^{\prime \prime \prime}: A^{\prime \prime \prime}[j][j]= \\
& \left.\quad A \operatorname{ssert}\left(\operatorname{Rankof}\left(\operatorname{Abs}\left(A^{\prime}[i][j]\right)\right)\right)<k t h\right)
\end{aligned}
$$

Here Assert() is like the function that we use in programming languages. It evaluates the enclosed boolean expression then returns 1 if the expression is true or 0 otherwise. Rankof() is for selecting feature coefficients to be quantized as 1 . This computation requires a sorting on coefficients in $A^{\prime}$. In the evaluation, transform domain coefficient with the largest magnitude has rank 1-st. A transform domain pixel whose rank is greater than $\mathrm{k}$ will become 0 , which represents a non-feature coefficient, in the feature plane $A^{\prime \prime \prime}$. Note that the result feature plane is binary and it, in fact, can be obtained from $A^{\prime \prime}$ by a nonstandard "quantization" in integer domain as the following.

$$
A^{\prime \prime \prime}: A^{\prime \prime \prime}[i][j]=\mid A^{\prime \prime}[i][j \mid
$$

After the process, those transform domain coefficients with larger magnitudes, no matter they are positive or negative, are kept as 1 . This speeds up the computation of distance measuring by our image metric $L^{S}$, which involves a boolean non-equal test and will be discussed in the following paragraphs.

\subsection{User Behaviors on Image Querying}

\subsubsection{Classifications of Query Behavior}

Consider if a subject without any training is asked for the first time to paint a query image, what the query image will be like? Correctly answering this question will have great influence on the design of our metrics.

Now we start to analyze the behavior of those who want to query for a particular image. One may paint or draw the query by looking at the printed or thumbnail version of that image. One can also do this from his impression of that image. In the former case, which is called painted-querying in tradition, one may investigate a thumbnail list of database images and select one from them that he wants to query for, or he may look at the printed image on books, on newspapers, or the oil paintings on wall. In the latter case, often called memoryquerying, one may have visited a fine art museum. Somewhat attracted by some painting, he may want to acquire a digital version after coming back home.

\subsubsection{Paint or Draw: How a Query Image is Pro- duced?}

Some researchers classified the querying behaviors into painted-querying and memory-querying, both kind of them were discussed in the previous section. They would often like to find different metrics for these two kinds of querying, ignoring the fact that the major difference lying behind the query behavior is his drawing or painting process instead of what he takes as his drawing reference. What we should argue here is that, in fact, no matter which kind of image one has used as his reference to paint his query, the key point is how he paint the query. That is, does he paint or draw the query? Or, does he draw with paint?

Do not be confused with the two words 'paint' and 'draw'. Here we just use the two words to classify the drawing behaviors. Painting a query means to use some or more colors to compose a figure. That is just what the impressionists do. Blurring the focus and emphasis on color distribution and on light presentation make the picture looks similar in different resolutions. On the contrary, drawing a query means to use a pen or pencil of one or a few colors(often the same color in various gray-scales) to sketch the borders or shapes of things in image. One may draw a query if he forgets the exact colors used in the picture to search for. Some people are color blinds or are just weak in distinguishing colors, which is exactly the case of the writer of this paper. Such kind of drawing query may help. Or sometimes there are images that are mainly composed of text. Drawing querying also plays a role for finding them.

Now the meaning of a 'draw with paint' query is thus well defined. This kind of query is the image that carries not only all or partial shape information but also all or partial color information that the user wants to express. Figure 1 demonstrates the two major kinds of queries classified by us.

Our purpose is to cope with all these situations. In our 
adjustable and dynamic metric $L^{P}$, the two extremes are $L^{S}$ and $L^{C}$. The $L^{P}$ can degenerate as $L^{S}$ or $L^{C}$ to meet the case that the query image is a pure drawn query or a pure painted one. It can also be a mixture of $L^{S}$ and $L^{C}$ by some percentage that can be used to deal with those drawn with paint' query images.

\subsection{Exploring the Proposed Metrics}

\subsubsection{The Adjustable Metric $L^{P}$}

In equation (1), $\lambda$ is just a floating value ranging from 0 to 1 that controls the percentage of the two solutes in our metric system, which are $L^{S}$ and $L^{C}$. When it is reset to 0 , the $L^{P}$ becomes $L^{C}$ and discriminates images only by the color distribution information of them. Conversely, if it is set to $1, L^{p}$ becomes $L^{S}$ and distinguishes images merely by the shape of the content objects.

Also, in our system, a user can adjust $\lambda$ to a percentage value whichever he wants between 0 and 1 if his query is 'drawn with paint'. In this case, the user can tune a slider bar, which represents current $\lambda$, to any percentage value depending on how much shape information his query image carries against color information.

\subsubsection{Color Metric $L^{C}$}

Now let's go further to the discussion of $L^{S}$ and $L^{C}$. Our $L^{C}$ is modified from $L^{Q}$, which also emphasizes on color distribution of query images. The major difference is on weight assignment.

Both in $L^{Q}$ and in our $L^{C}$, a specified weight is multiplied to the DC difference and other weights are used as multipliers to those differences on truncated, quantized coefficients. Conceptually, these weights will form a weight table. The weight table derived from the formula of $L^{Q}$, which is in level-of-pixel manner can be depicted in Figure 3. But here we assign the weights according to level-of-resolution criteria rather than level-of-pixel. Obviously, our $L^{C}$ uses the former but $L^{Q}$ is according to the latter.

\subsubsection{Level-of-Resolution Weight Assignment}

As Figure 4(a) shows, we should refresh the weight table of $L^{Q}$ with level of resolution shown in bold lines and re-assign values to the new table of $L^{C}$ by averaging the original pixel weights in the same block. In this scheme, pixels are gathered into blocks and blocks of the same resolution level are assigned equal weights. This makes sense. The relation between the weight table entry $w_{i, j}$ with respect to transform domain pixel location $(\mathrm{i}, \mathrm{j})$ and the weight that should be assigned to that table entry can be formulated in the following equation:

$$
\begin{aligned}
& W_{i, j}=W_{L n}, \text { where } \\
& n=\left\lfloor\log _{2}(\max (i, j))\right\rfloor
\end{aligned}
$$

We would not like to re-experiment on obtaining the weights again because the weight table of $L^{Q}$ has been experimented enough and proved to perform well. The result weight table of our $L^{C}$ is as Figure 4(b) demonstrates. So far, we get our metric $L^{C}$ from $L^{Q}$ by taking the same formula but assigning weights in different ways. What remains is the shape metric $L^{s}$ whose weight assignment is, surely, in level-of-resolution manner, too.

\subsubsection{The Novel Shape Metric $L^{S}$}

Matching shapes of image contents has been a problem in robot and machine vision for a long time. Most researchers try to solve this problem by finding edgedetection algorithms, which may include sharpening edges, applying filters, enhancing contrast, convex-hull linking, and so on. This probably misleads a few people. Some people might believe that the shape-matching problem is equal to the edge-detection problem and think that the only way for finding shape-like images is to detect the edges efficiently first.

If we were designing our metric $L^{S}$ by this way, it would not be so appropriate due to the extra computation time needed for edge detection and there is no time overlap between performing multiresolution signal processing for $L^{C}$ and performing edge detection for $L^{S}$. Thus we won't try to find the solution in this way. Again, we use the abstraction ability of multiresolution signal decomposition. We just benefit from those truncated, quantized transform domain coefficients that has been computed during previous processes and at the same time has been used by $L^{C}$ metric. This really utilizes the computation power and kills two birds with one stone.

\subsection{Inside $L^{S}$}

The idea of $L^{s}$ is simple from the equation. As we have mentioned, though the equation (2.2) for $L^{s}$ is like the right-hand-side of the operand ' $t$ ' in $L^{C}$, the two metrics are by no means the same. To compare the $L^{S}$ metric with $L^{C}$, consider the truncated and quantized coefficients $A$ " in equation (3) and equation (2.2), which was written so from the regular form (2.1) for comparison. Since there are only three levels in $A^{\prime \prime}$, only a few cases are possible for a given pixel. For clear realization of $L^{C}, L^{S}$ and their difference, let's consider a specified pixel location $(\mathrm{i}, \mathrm{j})$ on two associated transformed plane $A$ " and $B$ ". We can enumerate these cases and discuss how they will effect on the result distance computed by two metrics $L^{C}$ and $L^{S}$ :

(1) If $A^{\prime \prime}[i][j]=1$ and $B^{\prime \prime}[i][j]=1$ or $A^{\prime \prime}[i][j]=-1$ and 
$B "[i][j]=-1$ or $A^{\prime \prime}[i][j]=0$ or $B^{\prime \prime}[i][j]=0$, this pixel means the same thing on both planes and contributes nothing to both metric.

(2) If $A "[i][j]=1$ and $B "[i][j]=0$ or $A "[i][j]=0$ and $B^{\prime \prime}[i][j]=1$ or $A^{\prime \prime}[i][j]=-1$ and $B^{\prime \prime}[i][j]=0$ or $A^{\prime \prime}[i][j]=0$ and $B^{\prime \prime}[i][j]=-1$, then the pixel on one plane means there is a feature but that on another doesn't. In both metric, these points contributes to the distance exactly the weight value associated with its location on the weight table.

(3) We have discussed seven cases of the nine possible ones. There are still two cases possible and these cases makes the two metrics greatly different. Consider $A "[i][j]=1$ and $B "[i][j]=-1$ or $A^{\prime \prime}[i][j]=-1$ and $B^{\prime \prime}[i][j]=1$. In $L^{C}$, the absolute value of $A^{\prime \prime}[j][j]-B^{\prime \prime}[i][j]$ will be 2 . Such a pixel contributes double of the weight to the metric distance. This implies that there are much dissimilarities on the two planes at this location, because the 1 in one plane means a large positive transform domain value and the -1 in another means a very negative one. This makes sense for the metric $L^{C}$ from this point of view. But in $L^{S},\left|A^{\prime \prime}[i][j]\right|-\left|B^{\prime \prime}[i][j]\right|$ will become 0 , let alone the absolute value of it. This implies that, in $L^{S}$, this pixel means the same thing on both planes, instead of great dissimilarity. How this could happen??

If we inspect our signal decomposition process, which is the Haar transform, by a magnifying glass, we will find something surprising. This function, as we have mentioned, performs 1-D Haar decomposition on a sequence of discrete signals.

Suppose we are going to decompose a 1-D signal signal [] by Haar transform, we can see that, in fact, the transform can be divided into $\log _{2} S$ passes, depending on the signal size $S$. The first pass deals with the whole signal, repacks the signal, and then halves it into two parts. The second pass deals with the left-half that we are interested in, while the third does with the left-half of the lefthalf, whose size is a quarter of the original Signal [], and so forth. During each step in a pass, the algorithm just takes a pair of adjacent signal values, calculates their sum and difference, normalizes the results by a constant factor, and redistributes these values into two locations in two halves(or bands), to form a new signal.

The right half of the repacked signal stores the information about differences of nearby signal values. These values, in 2-D, hold high frequency information representing the edge appearances of the original image.

Turn back to our $L^{S}$ right now. Consider there is a transformed plane pixel truncated and quantized as 1 , and a pixel in the same location as -1 on another transformed plane. In our reasoning just discussed, at the specified location, there must be shape edges on both planes. Furthermore, there is exactly a negative gradient in the former plane and there is exactly a positive gradient in the latter. This implies that here, in $L^{s}$, we treat both positive and negative-gradient edges as the same. A pixel location with great positive value in one transformed plane but with great negative value in another should contribute nothing to $L^{s}$ metric!

In the $L^{S}$ metric, in fact, no matter the edge is of positive or negative gradient, it is merely 'an edge' from our point of view. This reflects the fact that there are only two meaningful quantization levels in $L^{S}$ but three in $L^{C}$.

Still two points worth noting are that we do not accumulate the DC difference to our shape distance measurement and that we only count on only Y-planes in $L^{S}$ metric. The first point is obvious since our ShapeMetric $L^{S}$ is a pure shape metric and it should not take any information about average intensities of each color channel. The second point is derived from the fact that human-sensible edges can be shown in gray-scaled Y-plane of an image and it, at the same time, saves a certain amount of computation time as well. Experimental results also shows that taking I, Q planes into account helps nothing. As we will see in the following chapters, our searching time is proportional to how much couples of $L^{S}$ and $L^{C}$ are computed, that is, how much database entries there are. While the growth of image database can not be avoided, this time saving on $L^{s}$ becomes extremely critical.

We shall give an illustration to show the query by shape in Figure 2. Here we draw a query image by its content shape and want to search for that image in our database. If we purely use our $L^{C}$ metric, which is a colorbased one improved from traditional $L^{Q}$, we will get nothing but that image whose color distribution is alike.

\subsection{Image Conversion Policy}

In our model, there are at least two requirements on images for 2-D multiresolution signal processing. First, the image should be square. Second, the width and height of the image should be power of 2 . Since not all of the collected images are both square and power-of- 2 sized, some conversion step must be taken before feeding image data into the Haar transform filter. To achieve this, different conversion strategies are adopted in our model for different components.

Area sampling has been a major technique used to prevent the aliasing effect[6][11]. In our query system, we would use the concept supported from area sampling. Suppose our source image size is $\mathrm{WxH}$, the sampled image size is $\mathrm{SxS}$. Then our $\mathrm{S}$ can be computed from:

$$
S=\operatorname{pow}\left(2,\left\lfloor\log _{2}(\min (W, H))\right\rfloor\right)
$$

Taking the advantage of that our area-sampled image size is 'the nearest 2 power size' of the original one, we have devised a fast algorithm for computing it.[13]

\section{A Snapshot of Our System}


The result is a system QueryStore II Plus built on Windows 95, as was shown in Figure 5. There are currently two components in the system, PowerIQ(Power Image Querying) for image database management with contentbased querying and SmartSCD(Smart Scene Change Detection) for video shot boundary detection. The PowerIQ not only supports normal primitives of image database manipulation, such as image registration, deletion, on-line browsing, listing, and modification, but is capable of content-based image querying either by shape, by color, or, by both. Moreover, it provides the user a free-foradjust metric and a comfortable query environment. Another component, SmartSCD, applies the shape metric and detects scene changes in video efficiently[1][2].

\section{IV.Experiments and Results}

\subsection{Metric System Parametrization}

There are several parameters controlling our metric system. The key parameter $\lambda$ of our metric $L^{P}$ can be adjusted dynamically while the system is running and should not be fixed. Thus our purpose is to look for the weight table WS for shape metric $L^{S}$ and the WC for color metric $L^{C}$. Besides, for both sub-metric, the number of topmost feature points to be considered(that is, how many transform domain coefficients with large magnitudes should be selected after quantization) for both submetric is also a problem. Here we are going to give only 6 different weights for each channel according to level of resolution(Figure 4(a)).

As we have mentioned, our $L^{C}$ is an improved version of $L^{Q}$, which takes 60 transform domain coefficients and its level-of-pixel weight assignment formula(by a function called bin()) is modified to form our WC weight table(Figure 4(b)). Our $L^{C}$ inherits its truncation(60 coefficients are considered) and quantization process (three levels) and uses our new level-of-resolution weight table. Thus our $L^{C}$ performs well for 'painted' queries that emphasize on color distributions and at least as well as $L^{Q}$ does. What follows is finding the parameters for our shape metric $L^{s}$.

\subsection{Derivation of the Weight Table for Metric $L^{S}$}

As we have discussed, the quantization process for $L^{s}$ results in only two quantization levels. Then we must find out how many coefficients left after truncation, which is an integer $t$, are proper for our $L^{S}$ metric and we should take an experiment for constructing the weight table WS for $L^{S}$

To make this experiment, 10 subjects were asked to 'draw' 30 shape-based query images and to indicate which image he was searching for. Then we start from $t=60$ to $t=300$ ( $t$ must greater than 60 because the nature of shape-based images), taking 10 as a step unit. For each t, we train the weight table WS by the concept of progressive refinement. For illustration, provided that we want to set up WS with its elements greater 0.0 and less than 20.0, and suppose we divide the range into four sub-ranges. We assign the weight of the multiresolution level 0 block(DC) in $\mathrm{WS}\left(\mathrm{WS}_{\mathrm{I} 0}\right)$ as 0.0 , level $1\left(\mathrm{WS}_{\mathrm{L} 1}\right), 2\left(\mathrm{WS}_{\mathrm{L} 2}\right), 3\left(\mathrm{WS}_{\mathrm{L} 3}\right)$, $4\left(\mathrm{WS}_{\mathrm{L} 4}\right)$ and $5\left(\mathrm{WS}_{\mathrm{LS}}\right)$ as 2.5 . Then we exhaustively iterate a 5-nested loop(which takes $4^{5}$ times) by incrementing $\mathrm{WS}_{\mathrm{L} 1}, \mathrm{WS}_{\mathrm{L} 2}, \ldots, \mathrm{WS}_{\mathrm{L} 5}$ by 5.0 in each loop, and evaluate the sum of rankings of the database images in relation to those queries'. Ideally, if all of the query images hit the topmost target(each time the first one in TargetView is exactly what the user searches for and the ranking of the image in that position is 1), the sum of their rankings will be 30 and this is the lower bound. After the above loops, suppose we get a best weight table whose 'the sum of rankings' is the smallest with $\left(\mathrm{WS}_{\mathrm{L} 0}, \mathrm{WS}_{\mathrm{L} 1}, \mathrm{WS}_{\mathrm{L} 2}, \mathrm{WS}_{\mathrm{L} 3}\right.$, $\left.\mathrm{WS}_{\mathrm{L} A}, \mathrm{WS}_{\mathrm{L} 5}\right)=(0.0,7.5,12.5,7.5,2.5,2.5)$. Then we iterate the 5-nested loop again starting with $\left(\mathrm{WS}_{\mathrm{L} 0}, \mathrm{WS}_{\mathrm{L} 1}\right.$, $\left.\mathrm{WS}_{\mathrm{L} 2}, \mathrm{WS}_{\mathrm{L} 3}, \mathrm{WS}_{\mathrm{L} 4}, \mathrm{WS}_{\mathrm{L} 5}\right)=(0.0,5.625,10.625,5.625$, $0.625,0.625)$. But this time the step amount is 1.25 to search for $\mathrm{WS}_{\mathrm{L} 1}$ in $[5.0,10.0], \mathrm{WS}_{\mathrm{L} 2}$ in $[10.0,15.0], \mathrm{WS}_{\mathrm{L} 3}$ in [5.0,10.0], and so on. Therefore we will find a better approximation of weight table WS by $\left(\mathrm{WS}_{\mathrm{L} 0}, \mathrm{WS}_{\mathrm{L} 1}, \mathrm{WS}_{\mathrm{L} 2}\right.$, $\mathrm{WS}_{\mathrm{L} 3}, \mathrm{WS}_{\mathrm{LA}}, \mathrm{WS}_{\mathrm{L} 5}$ ) again.

For each $t$, we find the best total ranking and the associated weight table by iterating the 5-nested loop three times ( 3 training depth). The value on weight table is thus of precision 0.3125 . As a result, we find that $t=110$ with the weight table shown in Figure 6 performs best.

\subsection{System Benchmark}

Our system was developed on Microsoft Windows 95 and can be executed from any PC. On such a platform, the amount of times needed for performing each of our processing steps individually were tested and summarized in Figure 7.

\subsection{Experimental Results}

For the validation of our metric system, 11 first-hand users were invited to paint 33 query images. He or she painted or drew their query images and at the same time answer our question "how much percentage of shape information does your query carry against color distribution information". We adjust our $L^{P}$ according to his answer and search against the database. The result is as Figure 8 shows. In this table, the reader can see that the query behavior is going to be toward the two extremes. 
That is, the query image either tends to emphasize on color distribution, or does on shape expression.

\section{Conclusion and Future Works}

\subsection{Conclusion and Discussion}

By making a survey on human behaviors of image querying, we have found that the major difference lying behind the query behavior is users' drawing or painting process. We have argued that the key point is how he produces the query. Thus we classify the query images into three main classes, drawn query, painted query, or both. To cope with all these situations, we devise an adjustable metric $L^{P}$ which is a mixture of the two metrics $L^{S}$ and $L^{C}$.

We have set up our color metric $L^{C}$ for dealing with those painted queries. We assign the weight values on our weight table in a reasonable 'level-of-resolution' manner. Since there is another existing metric $L^{\ell}$, also multiresolution-based but in 'level-of-pixel' manner, that had been well tested for painted queries, we decide to obtain our $L^{C}$ by reshuffling the weight table of $L^{Q}$ into an improved 'level-of-resolution' one.

Then we analyze those drawn query images and find that the key features of a drawn query are the shape edges. Unlike most previous approaches that is primarily by edge detection, we decide to match the shapes of two images in multiresolution transform domain. We also probe into the signal repacking process of wavelet decomposition and reveal that no matter the edge is of positive or negative gradient, it is merely 'an edge' from our point of view. Therefore we propose a shape metric $L^{S}$ with its two-level quantization feature extraction methodology. In the meanwhile, experiments by simulation are made to find out the proper weight table values for $L^{S}$, which are also organized in level-of-resolution, and to find proper numbers of coefficients to be kept as feature points.

Computation of each result metric takes just fractions of a second. Each $L^{S}$ measurement cost only 0.00084 second while $L^{C}$ is 0.00102 . Thus each $L^{P}$ takes 0.00187 in total to compute the distance between a pair of images. Therefore the metric computation time for searching against a 1000 -images database is merely 0.84 second for $L^{S}, 1.02$ for $L^{C}$, or 1.87 for $L^{P}$.

Yet another point worth mentioning is that the $L^{S}$ metric has been successfully applied to video scene change detection[1][2]. Unlike most previous detection operators, the $L^{s}$ metric not only detects scene change in multiresolution transform domain, but also avoids regarding some shape-invariant video effects, such as fade-out or flash, as scene changes. We have now integrated this part into our system.

\subsection{Future Work}

Here we list some topics for our future research:

Automatic Metric Adjustment. In our current system, the adjustable parameter $\lambda$ are free to adjust by the user. The user can adjust it if one is not satisfied with current querying result and can adjust it toward any possible direction. We are going to let our system automatically find a $\lambda$. To achieve this, several ideas may be considered. First, we could study the histogram of the query image. Therefore we would try to identify which class of query image does it belong to by the histogram. If the query image was a drawn one, often by two or a few high contrast colors, there would be only two or a few numbers of peaks in its histogram. We could classify the query image according to this clue. Alternatively, the system might also interact with the user each time before querying by bringing out the dialog for $\lambda$ adjustment. By this way, users could pre-set $\lambda$ by his feeling of how much shape against color information did his query image carry. Yet another way is to provide candidature querying. Each time before the querying result is shown, the system computes the querying results by pure $L^{S}$ and $L^{C}$. If either $L^{S}$ or $L^{C}$ is discriminate enough for current query image, the distances from the query image to the $1^{\text {st }}$-ranked target image and to the $2^{\text {nd }}$-ranked one should differ greatly. Thus we can select either $L^{S}$ or $L^{C}$ to be our default metric for this querying.

Further Metric Parameter Training. As we have indicated, the training depth of our current $L^{S}$ metric is 3 . That is, the precision of our current metric is 0.3125 . There are two ways to improve our weight table further. The first way is to train more deeply and get a better precision of our weight table. The second way is to consider more than one weight table in each training. As we have mentioned, we use 'the sum of rankings' to be our criteria for evaluating our weight table. We can set a threshold to filter these 'sum of rankings' and get more than only one weight table. A weight table that performs not so good in depth $n$ may perform well after training in depth $(n+1)$. That is, we give second chances to this kind of weight tables.

Finding Hardware Solution for $\boldsymbol{L}^{\boldsymbol{S}}$. As we can see, since the feature/non-feature characterization philosophy of $L^{S}$ is binary, the storage can be very small if we save the feature plane in our image database bit-wisely. Not only the feature plane extracted from the transformed plane are in binary form, but the computation of $L^{S}$ involves boolean non-equal tests. This implies that, $L^{S}$ distance measuring would be even faster and might be extremely fast if we have the dedicated hardware for computation. The hardware can, of course, take two bit-wise stored feature planes $A^{\prime \prime \prime}$ and $B^{\prime \prime \prime}$, perform the non-equal tests simultaneously, and output the binary results to another feature plane, say $C^{\prime \prime \prime}$. Then $C^{\prime \prime \prime}$ is used to mask another hard-wired $D^{\prime \prime \prime}$ plane, which represents the weight table. Taking an accumulation on the values of masked $D^{\prime \prime \prime}$ plane, we can obtain the distance easily. The entire process can surely be done in just a few clock cycles. Since the time overhead for transformation 
and quantization is fixed and the growth of the image database can not be avoided, this acceleration becomes critical. Total distance measuring time for searching against a database with 10,000 images by $L^{S}$ takes 8.4 second in current system but will be less than 1 second with hardware support.

\section{Acknowledgement}

We shall say special thanks to Chiou-Ting Xu, Kuang-Chih Lee, Herng-Yow Chen, and Jia-Qiang He for the original idea about video application, for the paper re-organization, for the suggestion of video processing, and for the article support.

\section{References}

[1] Zheng-Yun Zhuang, Chiou-Ting Hsu, Herng-Yow Chen, Ming Ouhyoung, Ja-Ling Wu, "Efficient Multiresolution Scene Change Detection by Wavelet Transformation", Proceeding of IEEE International Conference on Consumer Electronics 97, pp.250-251

[2] Zheng-Yun Zhuang, Tzong-Jer Yang, Ming Ouhyoung, "Multiresolution Scene Change Detection", submitted to IEEE Transactions on Consumer Electronics

[3] Charles E. Jacobs, Adam Finkelstein, and David H. Salesin, "Fast Multiresolution Image Querying" ACM SIGGRAPH'95 Conference Proceeding, pp. 277-286, 1995

[4] Shiuh-Sheng Yu, Jinn-Rong Liou, and Wen-Chin Chen, "Computational Similarity Based on Chromatic Barycenter Algorithm" IEEE Transactions on Consumer Electronics, Vol.42, No.2, MAY 1996, pp. 216-220

[5] John S. Boreczky and Lawrence A. Rowe, "Comparison of Video Shot Boundary Detection Techniques", Storage and Retrieval for Image and Video Database IV, SPIE 2670, pp. 170-199, 1996

[6] James D. Foley, Andries van Dam, Steven K. Feiner, and John F. Hughes, "Computer Graphics: Principles and Practice" Addison Wesley, 1993

[7] Ali N. Akansu and Richard A. Haddad, "Multiresolution Signal Decomposition, Transforms, Subbands and Wavelets", Academic Press, Inc, 1992

[8] Chiou-Ting Hsu and Ja-Ling Wu, "Multiresolution Mosaic," IEEE Trans. Consumer Electronics, vol. 42, no. 3, November 1996, pp. 981-990

[9] Rafael C. Gonzalez, and Richard E. Woods, "Digital Image Processing", Addison Wesley

[10] Alan V. Oppenheim, Ronald W. Schafer, "Discrete Time Signal Processing", Prentice Hall Inc, 1989

[11] Alan Watt, Mark Watt, "Advanced Animation and Rendering Techniques" ACM Press, Addison Wesley, 1993

[12] Adam Finkelstein, Charles E. Jacobs, and David H. Salesin, "Multiresolution Video" ACM SIGGRAPH'96 Conference Proceeding, pp. 281-290, 1996

[13] Zheng-Yun Zhuang, "Efficient Multiresolution Image Characterization Using Wavelet Transformation for Content-Based Image Retrieval", Master Thesis of National Taiwan University, 1997

[14] Alan L. Eliason, "System Development, Analysis, Design and Implementation", Harper Collins, 1990

[15] Chiou-Ting Hsu and Ja-Ling Wu, "Hidden Digital Watermarks in Images," under revised in IEEE Trans. Image Processing.
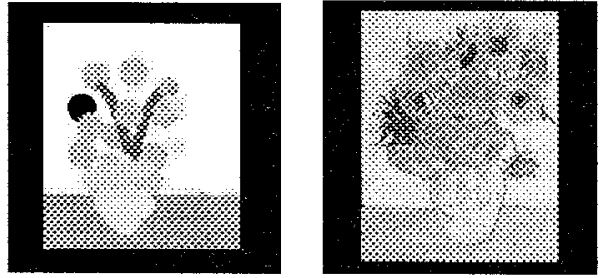

(a)
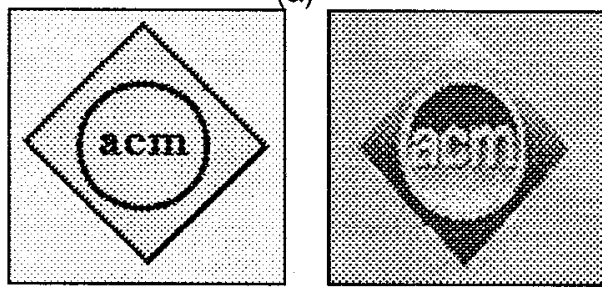

(b)

Figure 1: Two kinds of query images that a user tends to use. (a) a painted query image and its search target (b) a drawn query image and its target

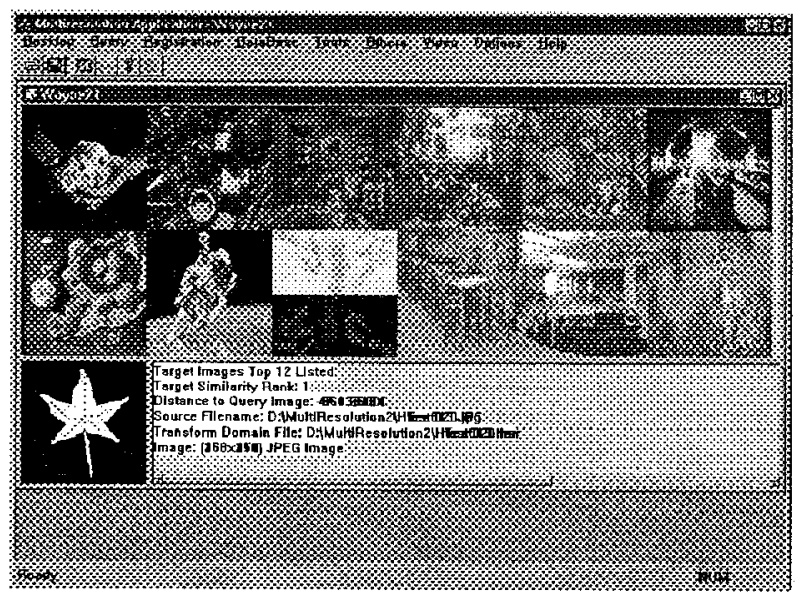

(a)

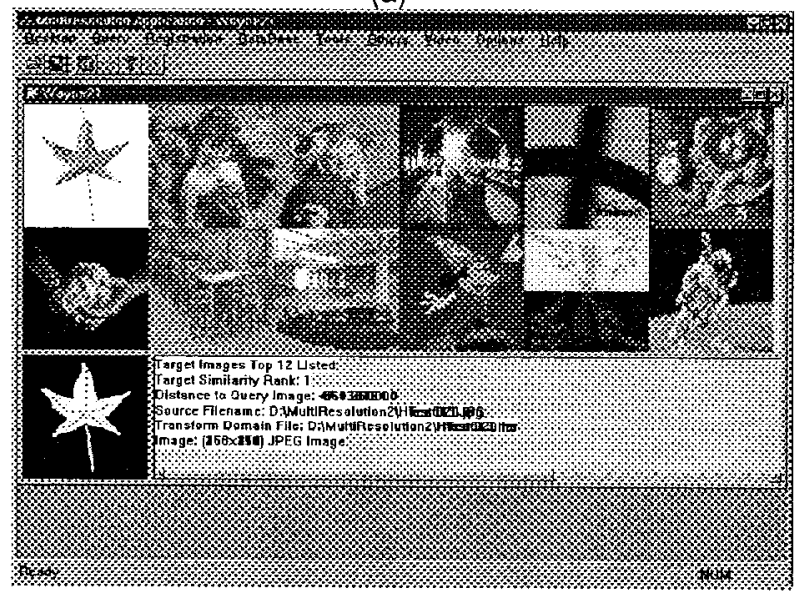

(b)

Figure 2: Search(bottom-left image) against our image database by a shape-based drawn query image (a) by color metric $L^{\circ}$, which shows incorrect result, which is listed in the topleft corner (b) by shape metric $L^{s}$, which shows correct result, top-left corner 


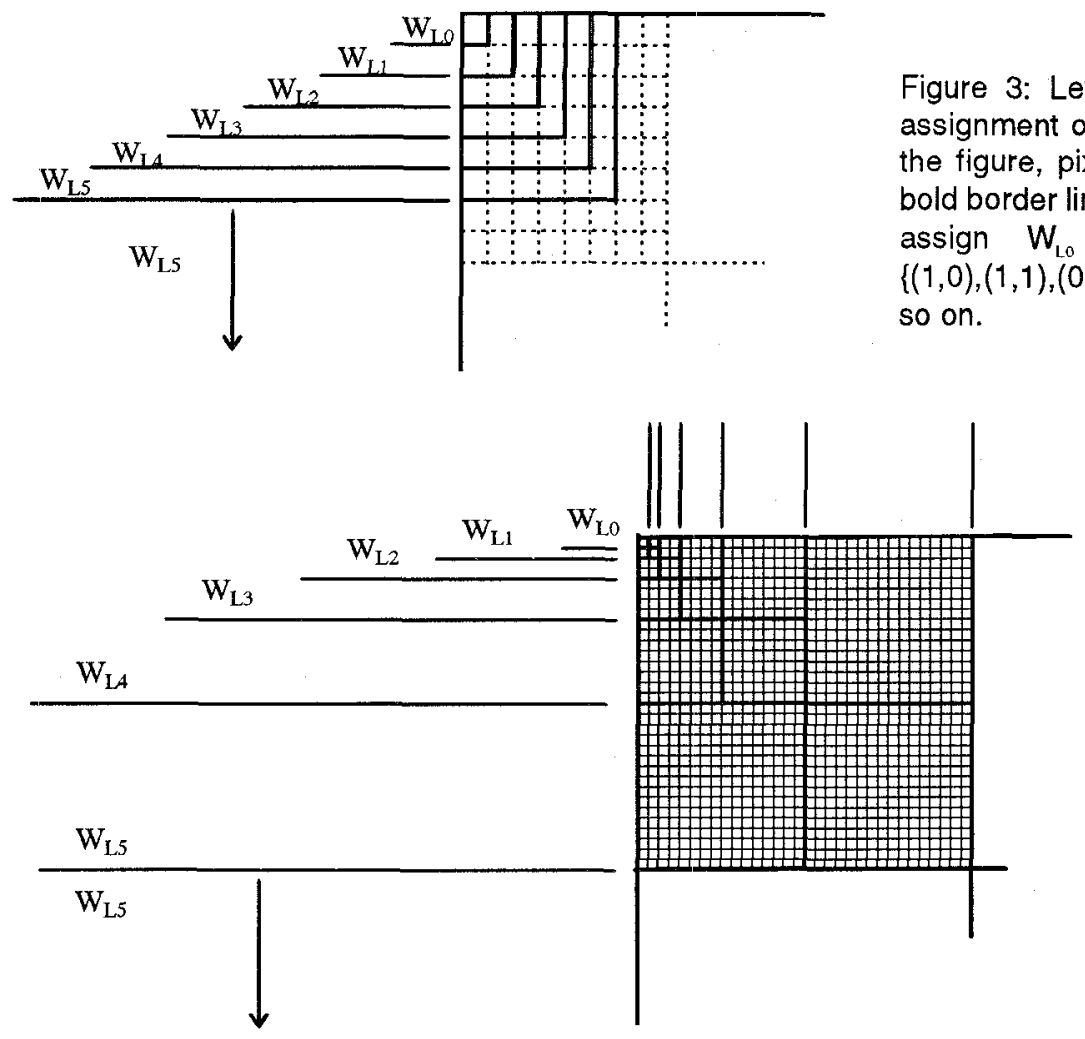

Figure 4(a): Assigning weights by the level of resolution method, blocks of pixels in the same resolution level are assigned equal weight; both our submetrics $L^{s}$ and $L^{c}$ apply this assignment rule

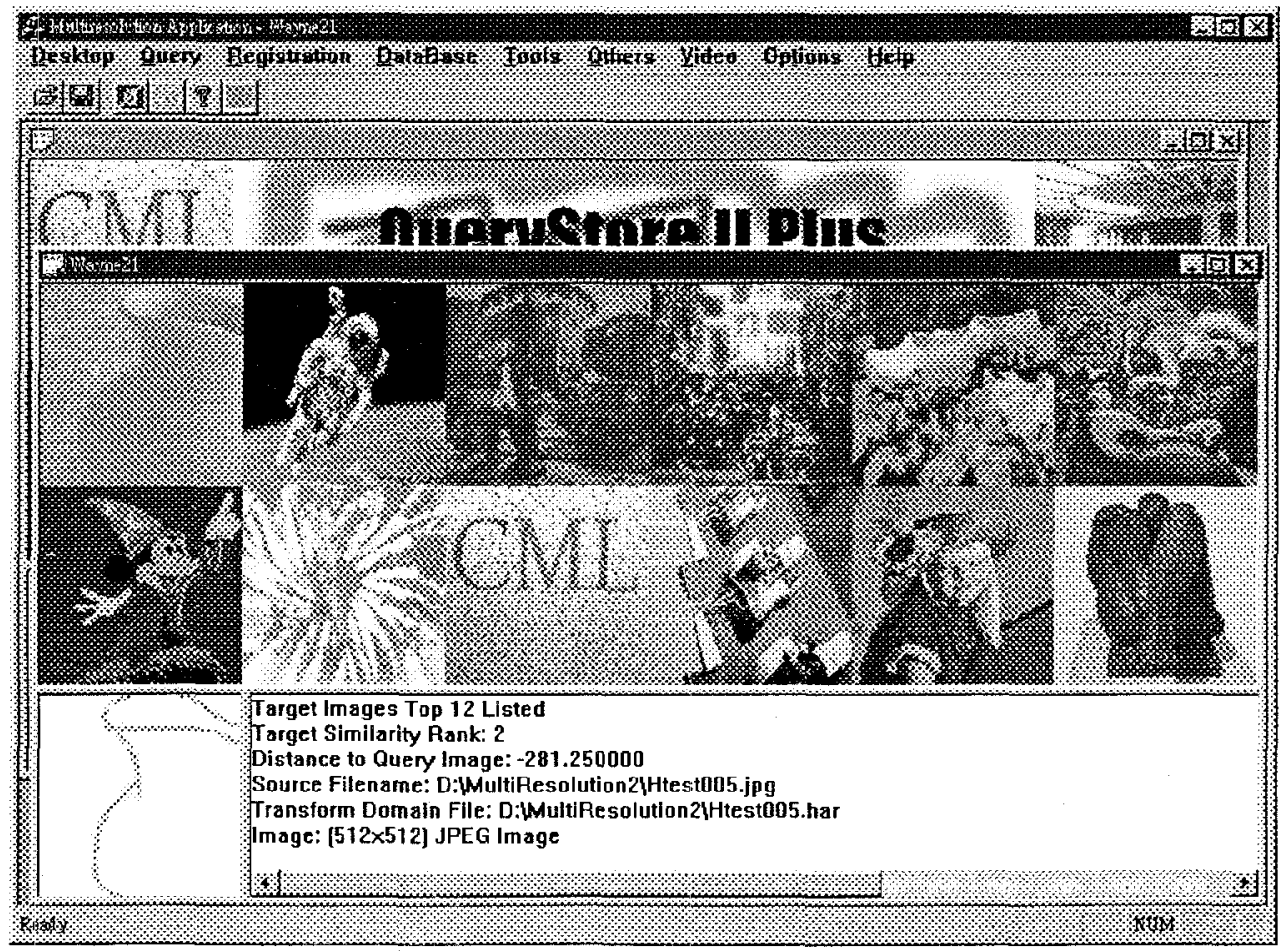

Figure 5: After we choose the 'Query by Content' item, the most contentsimilar target images will be browsed in TargetView(the upper window, in top left to lower right order) and the user can move the mouse around that window to get the information about that target image, which will appear in InfoView(the lower-right window).

The query image is shown in QueryView(the lower-left window).In this figure, we use a shapebased query image to query against the image 


\begin{tabular}{|c|c|c|c|c|c|c|}
\hline Domain & $W C_{10}$ & $W_{11}$ & $W_{C_{12}}$ & $W C_{13}$ & $W C_{14}$ & $W C_{15}$ \\
\hline$Y$ & 4.04 & 0.78 & 0.44 & 0.35 & 0.32 & 0.32 \\
\hline$I$ & 15.14 & 0.92 & 0.39 & 0.10 & 0.07 & 0.07 \\
\hline$Q$ & 22.62 & 0.40 & 0.44 & 0.24 & 0.38 & 0.38 \\
\hline
\end{tabular}

Figure 4(b): Values assigned to our WC weight table in level of resolution manner

\begin{tabular}{|c|c|c|c|c|c||}
\hline Domain & WS $_{14}$ & WS $_{12}$ & WS $_{13}$ & WS $_{14}$ & WS $_{15}$ \\
\hline$Y$ & 3.125 & 9.375 & 15.625 & 8.125 & 10.625 \\
\hline$I$ & N/A & N/A & N/A & N/A & N/A \\
\hline$Q$ & N/A & N/A & N/A & N/A & N/A \\
\hline
\end{tabular}

Figure 6: The weight table of our shape metric $L^{s}$ : weight values are assigned in 'level of resolution' style, which was shown in Figure 5(a); we only assign five weights here because DC values(level 0 transform domain pixels) are not considered by our $L^{s}$, WS, represents for the weight of level 1 coefficients, WS, does for level 2 , and so on

\begin{tabular}{|c|c|c|c|c|c|c|}
\hline Operation & Phase & Time(s) & $\mathrm{A}$ & $\mathrm{B}$ & $\mathrm{C}$ & Comment \\
\hline Image Registration & Total & 3.24 & $\bar{Y}$ & $Y$ & & Turnaround time \\
\hline Batch Query & Total & 3.84 & $\mathrm{Y}$ & $\mathrm{Y}$ & & Turnaround time \\
\hline \multirow{2}{*}{ Open Query Image } & Decompress JPEG file & 0.16 & $\mathrm{Y}$ & & $\mathrm{Y}$ & \\
\hline & Area Sampling & 0.27 & $\mathrm{Y}$ & & $\mathrm{Y}$ & \\
\hline \multirow[t]{2}{*}{ Preprocessing } & Transform to Long Floating & 0.17 & $\mathrm{Y}$ & & $\mathrm{Y}$ & \\
\hline & Transform to YIQ Domain & 0.11 & $\mathrm{Y}$ & & $\mathrm{Y}$ & \\
\hline \multirow[t]{2}{*}{ Wavelet Analysis } & Haar Transform & 1.32 & $\mathrm{Y}$ & & & 0.32 for a $128 \times 128$ image \\
\hline & Feature Extraction & 0.55 & $\mathrm{Y}$ & & & 0.22 for a $128 \times 128$ image \\
\hline \multirow[t]{7}{*}{ Querying } & Total Distance Measuring Time & 0.112 & & $\mathrm{Y}$ & & \\
\hline & \multirow[t]{3}{*}{ Individually for LS and LC } & 0.00084 & & & & $\begin{array}{l}\text { Compute the distance between a pair of } \\
\text { images by LS }\end{array}$ \\
\hline & & 0.00102 & & & & By LC \\
\hline & & 0.00001 & & & & Mix by LP \\
\hline & Tuple Retrieval & 0.058 & & $\mathrm{Y}$ & & \\
\hline & Ranking by Sorting & 0.00017 & & $\mathrm{Y}$ & & Quick sort by pointer \\
\hline & Candidate Image Browsing & 0.16 & & & $\mathrm{Y}$ & \\
\hline
\end{tabular}

Figure 7: System benchmark. This table shows the amount of time needed for each operation and step on our testing platform, an ordinary Pentium PC(133 Mhz). The unit of time is in second. This table also shows the properties of each step. For some step, if A property is 'yes', then the time is relative to the size of the query image. If B is 'yes', the time amount is effected by the size of current image database. If $\mathbf{C}$ is 'yes', that step is optional and may be omitted in some

\begin{tabular}{|c|c|c|c|c|c|c|c|c|c|c|c|c|}
\hline $\begin{array}{l}\text { User } \\
\text { Given } \lambda\end{array}$ & $0 \%$ & $10 \%$ & $20 \%$ & $30 \%$ & $40 \%$ & $50 \%$ & $60 \%$ & $70 \%$ & $80 \%$ & $90 \%$ & $100 \%$ & Total \\
\hline \#Queries & 3 & 1 & 4 & 1 & 1 & 4 & 0 & 2 & 5 & 2 & 10 & 33 \\
\hline $1^{\text {St }} \operatorname{Ran} k$ & 3 & 1 & 2 & 1 & 1 & 4 & 0 & 2 & 4 & 2 & 9 & 29 \\
\hline $2^{\text {na }} \operatorname{Rank}$ & 0 & 0 & 1 & 0 & 0 & 0 & 0 & 0 & 0 & 0 & 0 & 1 \\
\hline $3^{\text {rd }} \operatorname{Rank}$ & 0 & 0 & 1 & 0 & 0 & 0 & 0 & 0 & 1 & 0 & 0 & 2 \\
\hline $4^{\operatorname{tr}} \operatorname{Rank}$ & 0 & 0 & 0 & 0 & 0 & 0 & 0 & 0 & 0 & 0 & 1 & $I$ \\
\hline $5^{\mathrm{tr}} \sim$ & 0 & 0 & 0 & 0 & 0 & 0 & 0 & 0 & 0 & 0 & 0 & 0 \\
\hline
\end{tabular}

Figure 8: Experimental Result: this table shows the hit rate of our testing query images drawn by 11 first-hand users; they are invited to paint or draw 3 query images and then asked 'how much shape information does each of your query image carry, or how much alike in color distribution is your query image with the target image?' The test database has 60 images and the result shows that our $L^{\circ}$ has a hit rate up to $88 \%$ if the top one choice is evaluated, and becomes $97 \%$ if we consider top $5 \%$ of candidate queue as reasonable search targets. 